\title{
EL DESAFÍO CATALÁN. UN ANÁLISIS DESCRIPTIVO
}

\author{
THE CATALONIA CHALLENGE. A DESCRIPTIVE ANALYSIS
}

\author{
Elena Llorca Asensi \\ Universidad de Alicante. España/Spain \\ ellorca@yahoo.com
}

Recibido/Received: 12/04/2015

Modificado/Modified: 17/09/2015

Aceptado/Accepted: 29/09/2015

\section{RESUMEN}

El doce de diciembre de 2013 el Presidente de la Generalitat de Catalunya, Artur Mas, acompañado por representantes de algunas organizaciones de la vida política y social catalana, confirmó ante los medios de comunicación su decisión de llevar a cabo una consulta popular para conocer el respaldo de la ciudadanía a una posible independencia de Cataluña respecto de España. La fecha de la misma sería el 9 de noviembre de 2014. Este "desafío independentista" planteado por la Generalitat de Cataluña situó al Gobierno español en una situación harto difícil. En un clima de absoluta confrontación, en el que partidarios y detractores de la consulta habían dejado a un lado de facto la posibilidad de acuerdo, la brecha política y social abierta a raíz de este conflicto está hoy lejos de resolverse.

\section{PALABRAS CLAVE}

Cataluña, independencia, Estado, soberanía, secesión, referéndum, desafío.

\section{SUMARIO}

1. Introducción. 2. Objetivo del estudio. 3. Antecedentes: el catalanismo, el nacionalismo catalán y el independentismo. 4. La postura de Europa en el conflicto. 5. Los protagonistas del conflicto: partidos políticos y sociedad civil. 6. La opinión pública. 7. Propuestas o "vías" de resolución del conflicto. 8. La factibilidad de la consulta: aspectos técnicos, jurídicos y políticos. 9. Conclusiones. Bibliografía.

\begin{abstract}
On December 12th, 2013, the President of the Generalitat de Catalunya, Artur Mas, together with the representatives of some Catalan political and civil organizations, announced at a press conference its decision to conduct a referendum with the aim of stating the citizen's support for the independence of Catalonia from Spain. This referendum was to be held in November 9, 2014. This "independence challenge" posed by the Catalan government puts the Spanish government in a very difficult situation. In a climate of absolute confrontation, in which supporters and opponents of the consultation have put aside the possibility of a de facto agreement, political and social gaps opened in the wake of this conflict is far from being solved.
\end{abstract}

\section{KEYWORDS}

Catalonia, Independence, State, sovereignty, referendum, challenge.

\section{CONTENTS}

1. Introduction. 2. Goals of this study. 3. Backgrounds: Catalanism, Catalan nationalism and independentism. 4. The European position. 5. Starring the conflict: political parties and civil society. 6. The public opinion. 7. Possible "ways" to solve the conflict. 8. Feasibility of the consultation: technical, legal and political aspects. 9. Conclusions. References. 


\section{INTRODUCCIÓN}

El doce de diciembre de 2013 el Presidente de la Generalitat de Catalunya, Artur Mas, acompañado por representantes de distintas organizaciones de la vida política y social catalana, confirmó en rueda de prensa su decisión de llevar a cabo una consulta popular para conocer el respaldo de la ciudadanía a una posible independencia de Cataluña respecto de España. La fecha de dicha consulta sería el 9 de noviembre de 2014.

Este anuncio supuso un desafío público por parte del gobierno regional hacia el nacional. El Presidente Mas daba así por finalizado un largo período de desencuentros con el gobierno de Mariano Rajoy, un período en el que las reivindicaciones nacionalistas habían respetado, aunque a veces en el filo, el marco jurídico español, para pasar a una nueva etapa de rebelión en pro de la independencia de Cataluña.

En este movimiento independentista, que empezó hace años pero que ha ido paulatinamente cobrando fuerza y que formalmente arranca en solitario con esta comparecencia ante los medios, el Presidente de la Generalitat está acompañado por partidos políticos de corte independentista y por organizaciones de la sociedad civil catalana favorables a esta tendencia. Frente a ellos, el resto de partidos y organizaciones que rechazan la secesión y defienden la indisolubilidad del Estado.

\section{OBJETIVO DEL ESTUDIO}

El presente estudio realiza un análisis descriptivo de lo que se conoce como el "desafío catalán" en el momento presente, con objeto de contextualizarlo en el fenómeno del independentismo catalán y de describir las posiciones de los distintos gobiernos, partidos políticos y asociaciones civiles implicadas en el conflicto, a través de la investigación documental y de las declaraciones de todos ellos en distintos medios de comunicación el día doce de diciembre, día del "desafío", y fechas posteriores.

\section{ANTECEDENTES: EL CATALANISMO, EL NACIONALISMO CATALÁN Y EL INDEPENDENTISMO}

El catalanismo surge hacia 1830 como un movimiento cultural que reivindica la cultura y la lengua propia de la región, frente a la lengua y la cultura españolas. De sus postulados surge posteriormente, a principios del siglo XX, el nacionalismo catalán, como corriente que no sólo reivindica lengua y cultura propia, sino que además entiende que el territorio al que corresponde es un territorio separado de España, defendiendo por tanto la existencia de una nación catalana.

Así, el nacionalismo catalán llega a sostener no sólo que la cultura catalana es única y muy diferente de la española, sino que Cataluña es una nación oprimida por España desde su ocupación por las tropas borbónicas en 1714, especialmente tras la promulgación de los Decretos de Nueva Planta de Felipe V que, buscando la unificación de los distintos territorios de los que era soberano, suprimió con estos decretos las instituciones catalanas y estableció el castellano como lengua oficial a utilizar en la administración. Debe subrayarse que estas medidas fueron adoptadas no sólo en Cataluña, sino en el resto de territorios que formaban la Corona de Aragón: el Reino de Valencia, el Reino de Aragón y el Reino de Mallorca, así 
como en la Corona de Castilla. Según el historiador Ricardo García Cárcel, la aplicación de los Decretos de Nueva Planta supuso el "triunfo de la España vertical sobre la España horizontal de los Austrias" (García Cárcel, 2002). La España horizontal era la que planteaba la realidad nacional como un agregado de territorios y una identidad española plural, mientras que la vertical es la "España centralizada, articulada en torno a un eje central, que ha sido siempre Castilla, vertebrada desde una espina dorsal, con un concepto de una identidad española homogeneizada» (García Cárcel, 2002: 114).

Pero un paso más dentro de esta corriente es el llamado Independentismo catalán, que se reafirma en los postulados anteriores y, además, promueve la secesión de Cataluña en un Estado independiente, plenamente soberano y capacitado para integrarse en la Unión Europea como un Estado más. La corriente más extrema del independentismo catalán vas aún más allá y pretende la integración en este nuevo Estado no sólo de Cataluña, sino de varios territorios bautizados como "Países Catalanes": La propia Cataluña; el Rosellón y la Cerdaña franceses (a los que denominan Cataluña Norte); la Franja Oriental de Aragón a la que denominan Franja de Ponent y consideran también parte de Cataluña; la Comunidad Valenciana; las Islas Baleares; la comarca murciana de El Carche y la ciudad sarda de Alguer, en Italia. Todos estos territorios, a los que se uniría el Principado de Andorra, formarían, tras independizarse, un Estado confederado catalán.

\section{LA POSTURA DE EUROPA EN EL CONFLICTO}

Tal como se indica en la Introducción, el gobierno catalán decidió, en vista de que sus reivindicaciones no eran atendidas, emprender en solitario y enfrentándose al gobierno central de España el camino hacia la independencia de esta Comunidad Autónoma.

El Presidente del gobierno, Mariano Rajoy, estaba, en el momento del anuncio de Mas, reunido con el Presidente del Consejo Europeo Van Rompuy. Al finalizar la reunión, y tras informar del resultado de la misma, Rajoy abordó el tema reafirmándose en su postura acerca del desafío y, así, anunció que su gobierno "no puede ni autorizar ni negociar sobre algo que es propiedad de todos los españoles" (ver en la bibliografia la comparecencia de Mariano Rajoy ante los medios el 12 de diciembre de 2013, min 0:32). Ese "algo" es la soberanía nacional que, como indica la Constitución Española en su artículo 1.2. "reside en el pueblo español, del que emanan los poderes del Estado".

En esta comparecencia, el presidente español fue apoyado en sus declaraciones por el Presidente del Consejo Europeo, Herman Van Rompuy, quien confirmó, a preguntas de los periodistas, que cualquier territorio que se separe de un país de la Unión Europea deja formar parte de la misma ya que los Tratados de la Unión ya no se aplicarían a dicho territorio. Van Rompuy reforzó así al ejecutivo de Mariano Rajoy, añadiendo su deseo de que España permanezca como un país unido, ya que "estar unido es la mejor opción para todos los ciudadanos". Apuntó el Presidente del Consejo Europeo, además, que el paro en España estaba descendiendo, que la economía repuntaba y que los "valientes esfuerzos" realizados en los últimos tiempos estaban dando sus resultados. La postura de Europa en el conflicto quedó clara: no lo apoyan, ni lo desean. 


\section{LOS PROTAGONISTAS: PARTIDOS POLÍTICOS Y SOCIEDAD CIVIL}

No sólo los presentes en la rueda de prensa, sino aquellos ausentes por voluntad propia e incluso los no invitados, quedaron retratados como protagonistas de este conflicto. Junto a Artur Mas en su comparecencia se situaban Ramón Espadaler, Conseller y presidente del Consell Nacional de Unió; Jordi Turrull, Portavoz del Parlamento Catalán; Joana Ortega, Vicepresidenta de la Generalitat; Artur Mas (CiU), Presidente de la Generalitat de Cataluña; Oriol Junqueras, líder de ERC (Esquerra Republicana de Catalunya); Joan Herrera (ICV EUiA); Joan Mena (EUiA); David Fernández (CUP - Candidatura d'Unitat Popular). Así pues: gobierno, parlamento y partidos de corte independentista.

Veamos a continuación las posiciones de todos los protagonistas del conflicto, tanto a favor como en contra de realizar el referéndum por la independencia de Cataluña:

\subsection{A favor de realizar una consulta}

Se sitúan en este eje aquellas organizaciones que defienden el derecho de una parte del país a decidir si quiere seguir perteneciendo al mismo. La gran mayoría persigue, además, la independencia, pero hay casos en que, aun no deseando la independencia, sí se defiende el derecho de esa parte de los ciudadanos a ser consultados (PSC o CDC).

\section{Partidos Políticos:}

- CiU: Convergencia i Unió, partido presidido por Artur Mas. El partido se forma como federación entre Convergencia Democrática de Cataluña y Unión Democrática de Cataluña. CiU ha estado en el gobierno catalán desde 1980 hasta la actualidad, con un paréntesis de 7 años en que una alianza entre partidos de izquierda (el conocido informalmente como tripartito- Alianza entre PSC, ERC e ICV) les quitó el gobierno. De tradición demócrata cristiana, se define como un partido catalanista y, desde la subida de Artur Mas a la presidencia, marcadamente independentista. A pesar de su carácter regional, CiU ha tenido presencia nacional al colaborar tanto con el PP como con el PSOE, prestando su apoyo para que éstos pudieran formar gobierno en momentos en que no habían obtenido suficiente mayoría.

Es interesante desglosar por un momento a Convergencia Democrática de Cataluña (CDC) de la federación que acabamos de mencionar (CiU) para resaltar que su secretario general, Josep Antoni Durán i Lleida, aunque apoya el derecho a la autodeterminación, es el defensor de la "tercera vía" que, según veremos más adelante, defiende una Cataluña federada o no - independiente de España, haciendo hincapié en que el referéndum propuesto por sus socios en $\mathrm{CiU}$ y el resto de partidos independentistas es meramente consultivo y no vinculante. Prefiere el federalismo a la independencia aunque no descartaría ésta si es la voluntad del pueblo catalán. En todo caso, no la quiere sin el acuerdo de España (RAC1: $12126 \mathrm{H}$ min 51:10), de lo que se deduce lógicamente la existencia de tensiones en torno a esta cuestión central, dentro de dicha federación de partidos.

- PSC: Partido Socialista de Cataluña. Rama regional del PSOE, principal partido de la oposición en España. Está a favor de realizar una consulta, siempre y cuando sea acordada con el Gobierno. Se opone al desafío de Más y sus socios del frente separatista. No obstante, el 16 de enero de 2014 - pocos días después del anuncio de Mas - el Parlamento Catalán aprobó iniciar un trámite para solicitar al gobierno central la celebración del referéndum, y 3 parlamentarios de PSC rompen la disciplina de partido votando sí a dicha solicitud, lo que fue considerado por varios medios de comunicación como la "ruptura del Partido Socialista en Cataluña". 
- ERC: Esquerra Republicana de Catalunya, partido de izquierdas, antimonárquico, independentista. Aboga por la independencia de lo que se conoce como "Países Catalanes", es decir, es parte de esa facción más extrema del independentismo catalán. El líder de ERC, Oriol Junqueras, ha manifestado en varias ocasiones que, si el Estado no permite hacer la consulta, desde su partido llamarán a la movilización y la llevarán igualmente a cabo. No descarta asimismo Junqueras la declaración unilateral de independencia en caso de que la consulta resultara favorable.

- ICV - EuiA: coalición entre Iniciativa por Cataluña Verdes e Izquierda Unida y Alternativa, de carácter ecologista e ideología de izquierdas.

- CUP - Candidatura de Unidad Popular. Organización política asamblearia, catalana, de izquierda e independentista que data de 1987. Defiende, como ERC, la independencia de los "Países Catalanes".

Otras organizaciones políticas de la izquierda independentista como Endavant (que significa "en adelante"), el Movimiento en Defensa de la Tierra (MDT) o el Partido Socialista de Liberación Nacional (PSAN por sus siglas en catalán) forman parte también del bloque político favorable al independentismo.

\section{Representantes de la sociedad civil:}

- Asamblea Nacional Catalana: organización civil pro-independentista, de reciente constitución -apenas el 10 de marzo de 2012. Se trata probablemente de la organización más beligerante a favor de la secesión, siendo su objetivo único definir y recorrer la hoja de ruta hacia la independencia. No confundir con la Asamblea de Cataluña, organización que reunió a toda la oposición antifranquista catalana desde su creación en 1971 hasta su disolución, por diferencias políticas, en las primeras elecciones generales democráticas de 1977. La Asamblea Nacional Catalana es la asociación emblema de la independencia y su presidenta, Carmen Forcadell, ha sido bautizada en los medios como "la guardiana del independentismo".

Por otra parte, entre las organizaciones juveniles especialmente activas en la búsqueda de la independencia podríamos mencionar a Juventud Nacionalista de Cataluña, la organización y Arran o el Sindicato de Estudiantes de los Países Catalanes (SEPC). Asimismo, existen multitud de otras organizaciones de izquierda que apoyan el movimiento independentista, sin que sea dicho movimiento el eje central de su actuación. Se trata normalmente de organizaciones "anti sistema", que incluyen entre sus postulados la defensa de la autodeterminación. Podemos destacar, entre muchas otras: Corriente Roja (se autodefine como de "carácter marxista, de lucha anticapitalista, de clase, radicalmente democrática, internacionalista, ecologista y feminista"), Lucha Internacionalista, En lucha, Revolta global, Universidad Comunista de los Países Catalanes, L’Accent (periódico popular de los Países Catalanes), etc.

Por último, existen incontables organizaciones, casales (comunidades catalanas en el exterior, que han jugado un gran papel en la difusión de la cultura catalana), centros okupados e iniciativas de la Izquierda Independentista a nivel local.

\subsection{En contra de realizar una consulta}

Las posturas a este lado del conflicto defienden que el Estado es una unidad indisoluble o bien que la soberanía del mismo reside en todos los ciudadanos españoles y no sólo en una parte de ellos, por lo que, en última instancia, habría que convocar un referéndum nacional 
para ver si los españoles renuncian a una parte de su país y no uno regional para preguntar a esa parte si quiere irse o quedarse.

Partidos políticos:

- Partido Popular: uno de los dos partidos mayoritarios en España (caracterizada tradicionalmente por un bipartidismo incompleto), que ocupa el gobierno en el momento de realizar el presente artículo, con Mariano Rajoy a la cabeza. Es radicalmente opuesto a la realización de una consulta y por supuesto a los principios separatistas de la denominada "Vía Catalana". Considera que el movimiento pro-consulta responde a la manipulación política. Concretamente, la líder del PP en Catalunya, Alicia Sánchez-Camacho señaló que, con su comparecencia ante los medios ese 12 de diciembre, que el Presidente Mas había "dinamitado todos los puentes de comunicación con el gobierno de España" (ver artículo de "El Periódico" en la bibliografía). El Ministro del Interior Jorge Fernández Díaz lo calificó como "un error histórico" (RAC1: “1212 16h Versió RAC1” min 14:30 y 15:29) que iba a provocar, sobretodo, una gran fractura social. Por su parte, el entonces Ministro de Justicia, Alberto Ruiz Gallardón, aseguró que la consulta "no se va a producir" porque es "ilegal, contrario a la Constitución y contrario al Estado de las Autonomías" (RAC1: "1212 16h Versió RAC1" min 12:50).

- PSOE: Partido Socialista Obrero Español, principal partido de la oposición, está en contra de la consulta anunciada por Mas. Su facción regional, sin embargo, la apoyaría siempre que no se haga sin acuerdo del gobierno. En palabras de su líder nacional Alfredo Pérez Rubalcaba, "el Presidente Mas está llevando a Cataluña a un callejón sin salida" (RAC1: "1212 16h Versió RAC1" min 25:45) y manifiesta sin tapujos su apoyo al Gobierno en esta cuestión.

- UPyD: Unión Progreso y Democracia, partido político que irrumpe en 2007 en el panorama político español, tradicionalmente polarizado entre las dos opciones anteriores, y que va ganando adeptos en los últimos años. Surge como una escisión del PSOE y se define como centro progresista, no nacionalista, laico y defensor de un modelo federalista en España y en Europa. En 2013 es liderado por Rosa Díez. Se opone a la consulta, por considerar que la soberanía del Estado reside en todos los ciudadanos y no puede una parte de los mismos decidir sobre la integridad del territorio de manera unilateral, llegando a considerar que el anuncio de los partidos independentistas "esconde cobardía, intención de manipular y ridículo" (RAC1: "1212 16h Versió RAC1" min 23:50). Hay que señalar que UPyD fue el primer partido en sugerir la posibilidad de intervenir la Autonomía de Catalunya (Crónica Global, 2014) a través del artículo 155 de la Constitución Española, lo que podría significar incluso la suspensión de los poderes conferidos al gobierno catalán, algo inédito en casi 40 años de democracia (De la Nuez, 2012). El Artículo 155 de la Constitución Española dice concretamente que: "1. Si una Comunidad Autónoma no cumpliere las obligaciones que la Constitución u otras Leyes le impongan, o actuare de forma que atente gravemente al interés general de España, el Gobierno, previo requerimiento al Presidente de la Comunidad Autónoma y, en el caso de no ser atendido, con la aprobación por mayoría absoluta del Senado, podrá adoptar las medidas necesarias para obligar a aquélla al cumplimiento forzoso de dichas obligaciones o para la protección del mencionado interés general. 2. Para la ejecución de las medidas previstas en el apartado anterior, el Gobierno podrá dar instrucciones a todas las autoridades de las Comunidades Autónomas.",

- Ciutadans: partido político cuya denominación completa es Ciudadanos-Partido de la Ciudadanía. De reciente creación, surge en 2006 a partir de la plataforma cívica Ciutadans de Catalunya y se define como un partido constitucionalista, no nacionalista y de centro izquierda. Su origen está en la oposición a lo que llaman el "nacionalismo obligatorio" 
imperante en la política catalana, y está formado inicialmente por intelectuales y profesionales agrupados en la plataforma cívica del mismo nombre. Su ámbito de actuación es nacional, pero su presencia real es mayoritariamente en Catalunya. Su Secretario General, Matías Alonso, declara tras el anuncio de Mas que "no respetar de forma escrupulosa el marco del estado de derecho no es en ningún caso democrático" (RAC1: "1212 16h Versió RAC1" min 34:58).

La Sociedad civil:

- Ciutadans de Catalunya: plataforma cívica y cultural formada por ciudadanos intelectuales y profesionales - que se oponen, desde una ideología de centro izquierda, al nacionalismo catalán.

- Convivencia Cívica Catalana: entidad creada en 1998 como reacción a la Ley 1/1998, de 8 de enero, de Política Lingüística aprobada por el Parlamento de Cataluña. Actualmente su actividad gira en torno a cuatro campañas, la "Campaña contra la politización de la escuela", la "Campaña por el bilingüismo en las escuelas", "Campaña contra las multas lingüísticas y la "Campaña por la bandera española".

\section{LA OPINIÓN PÚBLICA}

Entendemos por opinión pública la que corresponde a los ciudadanos, no manifestada a través de su voto o su integración en organizaciones civiles. Para conocer dicha opinión haremos un repaso por los resultados de las distintas encuestas o sondeos realizados sobre el particular. Vaya por delante que, dada la enorme trascendencia política de esta cuestión, los estudios estarán normalmente sesgados hacia el organismo que los haya encargado y/o pagado.

No obstante, antes de dar comienzo al análisis de las encuestas y sondeos de opinión realizados en relación al sentimiento nacionalista y a la independencia de Cataluña, merece la pena resaltar la correlación que parece existir entre las crisis económicas y el auge de sentimientos nacionalistas, nacionalismos excluyentes y xenofobia (OIM, 2010: 149). Desde este punto de vista, el agravamiento de la crisis económica española e internacional podría por tanto explicar, no en su totalidad pero sí en parte, la extensión de la demanda secesionista entre la población de la autonomía catalana. Así, diversos estudios permiten afirmar no sólo lo anterior sino que "las crisis económicas suponen una vulnerabilidad clara de los sistemas democráticos" (Aldeguer, 2013: 14). La opinión pública es más proclive al proteccionismo y a replegarse sobre sí misma cuando la economía es débil que cuando es fuerte. De ahí, en parte, que las encuestas reflejen cada año un porcentaje mayor de apoyo al proyecto independentista.

Un sondeo realizado el mismo día del anuncio de Mas, 12 de diciembre de 2013, indicaba que "El 44\% de catalanes votarían por la independencia y el 36\%, en contra" (Rico, 2013). Esta encuesta, una de las más difundidas por los distintos medios, fue encargada por $\mathrm{El}$ Periódico a dicho Gabinete. El Periódico de Catalunya es considerado uno de los medios afines al proyecto independentista y al gobierno de Mas, del que recibe cuantiosas subvenciones (Subvenciones de la Generalitat al Grupo Z (El Periódico): 600.000 euros en 2011, 946.000 euros en 2012. En total: 1.548 .000 euros). Más adelante volveremos sobre los resultados de esta encuesta.

Anteriormente, distintos organismos y centros de investigación realizaron también estudios para conocer cuál sería la respuesta de la ciudadanía. Citaremos aquí unos cuantos 
no sin antes subrayar que, para otorgar una mayor o menor credibilidad a los resultados obtenidos por cada uno de ellos sería necesario realizar un estudio específico sobre la metodología empleada, el tratamiento de los datos y la interpretación de los mismos. Dado que no es posible tener acceso a los datos en bruto de los sondeos, ni a su metodología detallada, no podemos más que señalar los resultados obtenidos, no porque los consideremos válidos (ni por exceso ni por defecto), sino porque sirven para ilustrar la información que ha circulado entre la ciudadanía (lógicamente, cada nuevo sondeo ha hecho correr ríos de tinta en los medios de comunicación):

- El Centro de Estudios de Opinión de la Generalitat de Cataluña CEO (organismo público adscrito a la Consellería de Presidencia) pregunta desde 2011 sobre el deseo de la independencia de la población, y desde esa fecha hasta sus últimas oleadas de 2013 registra un incremento de respuesta al "Sí, a la independencia" desde el 42,9 \% inicial hasta el 55,6 \% actual.

- La Universidad Abierta de Cataluña, por su parte, encarga a Instituto DYM una encuesta que en 2009 da un 50,3 de síes a la independencia, mientras que el CIS en 2001 da un 48,1\%. El ICPS, por su parte, indica que la tendencia independentista disminuyó desde el $35 \%$ de 1991 hasta el 31,7 de 2007. Es importante resaltar que la utilización de distintas metodologías da como resultado porcentajes de respuesta positiva muy diferentes, con diferencias de casi 10 puntos por encima si la entrevista es telefónica, frente a entrevista presencial (de ahí que intuir la fiabilidad de los datos sea una tarea harto compleja, como se apunta al inicio de este apartado).

En todo caso, y provengan de donde provengan los estudios, podemos ver que no se trata de un porcentaje pequeño de la población el que apoya la independencia. Los resultados más conservadores rondarían el 30\% y los más optimistas el 50\%.

Este análisis coincide de cierta forma con el realizado por Convivencia Cívica Catalana, que indica en su web: "Convivencia Cívica Catalana estima que la cifra de partidarios de la secesión en las encuestas del gobierno catalán está inflada, como mínimo, en un $20 \%$ debido al notable sesgo de la muestra que se ha seleccionado". Este sesgo es el resultado, según la misma organización, de una selección no aleatoria de la muestra de encuestados. CCC se refiere en general a todas las encuestas sobre el tema realizadas por el gobierno, pero realiza además un análisis específicos del Barómetro de Opinión Política, 3ra Oleada, del 22 de noviembre de 2013, publicado por el CEO (Centro Estudios de Opinión de la Generalitat de Catalunya). Respecto a dicho Barómetro, indica el CCC, “en la muestra del gobierno catalán están notablemente infrarrepresentados los votantes de partidos no nacionalistas. En concreto, se incluye un 70\% menos de votantes del PP (es decir, menos de una tercera parte que en la realidad), un 43\% menos de C's y un 15\% menos del PSC. Por el contrario, los votantes de opciones nacionalistas están considerablemente sobrerrepresentados: hay un 30\% más de votantes de CiU que en la realidad, un $47 \%$ más de ERC y un $10 \%$ más de la CUP” (CCC, 2013: 3).

Además de denunciar el sesgo de las encuestas desde el punto de vista metodológico, CCC denuncia también el importante sesgo político e ideológico con el que, en su opinión, carga el CEO, y lo hace de la siguiente manera: "Ello (referido al incorrecto muestreo) se une a la polémica que rodea al director del CEO, Jordi Argelaguet, nombrado por Artur Mas para el cargo a los pocos días de llegar a la presidencia del gobierno catalán y conocido por sus ideas políticas radicales. Argelaguet, anterior militante del Moviment de Defensa de la Terra (MDT), brazo político de la organización terrorista Terra Lliure, se ha manifestado públicamente partidario de saltarse la legalidad y la Constitución Española y ha calificado a España como "país vecino". Nos preguntamos si el actual director del CEO es la persona más 
idónea y objetiva para realizar encuestas sobre una hipotética secesión de Cataluña" (CCC, 2013: 4).

Tal como señala el CCC en las conclusiones de este análisis: "Desde hace mucho tiempo los políticos han descubierto que las encuestas no sólo miden el estado de opinión de una sociedad, sino que afectan a la opinión de la propia sociedad que está siendo medida. Por ejemplo, la publicación de información positiva sobre las intenciones de voto de un candidato o sobre el apoyo a una determinada idea política incide positivamente en la decisión de la población para simpatizar con ese candidato o con esa idea" (CCC, 2013: 27).

En este sentido, y a pesar del objetivo meramente descriptivo de este estudio, creo conveniente señalar que, en opinión de la autora del mismo, la manipulación de las encuestas -y consecuentemente de la opinión pública- existe (a pesar de que no hay estudios concluyentes que midan el peso de dicha influencia en la población) y no es una actividad que afecte únicamente a los independentistas. Antes bien, es una actividad frecuentemente realizada desde las esferas de poder, sean estas políticas, económicas o culturales, y por ello no puedo estar más de acuerdo en la afirmación de CCC citada en el párrafo anterior (aunque probablemente sin la intención de que esta conclusión les pueda señalar también a ellos mismos).

Como hemos señalado al abrir este apartado, el Gabinete de Estudios de Opinión Pública, GESOP, realizó el mismo día del anuncio de Mas una encuesta para saber cuál sería la respuesta a la doble pregunta planteada. Más allá de los resultados, publicados en portada de El Periódico, el GESOP mismo advierte que "hay que tener presente que estas cifras no son una proyección o una estimación del resultado que tendría la consulta si se celebrase hoy. Para que eso fuera así, se tendrían que establecer factores de corrección a partir de otras variables para atribuir una estimación final a cada una de las opciones". Lo que quiere decir este centro tiene que ver con lo expuesto por el CCC respecto a la encuesta del CEO: si no se garantiza que la muestra encuestada representa fielmente a la sociedad catalana, los resultados de estudio carecen de validez. El Periódico, no obstante, no parece interesado en esta reflexión cuando publica en portada el titular: "El 44\% de catalanes votarían por la independencia y el $36 \%$, en contra".

\section{LAS DIFERENTES PROPUESTAS O "VÍAS" DE RESOLUCIÓN DEL CONFLICTO}

\subsection{La vía de la unidad o indisolubilidad del Estado}

Es la postura defendida por los partidos mayoritarios (PP y PSOE) así como por UPyD (nacional) y Ciutadans. Se oponen frontalmente a la consulta y, por supuesto, a la secesión de una parte del Estado, aunque pudieran abrir la puerta a un mayor grado de autogestión e incluso a hablar de federalismo. Para ello, argumentan que sólo el Estado en su conjunto podría ser consultado sobre una hipotética secesión de una parte de sí mismo, pues la soberanía pertenece al conjunto y no a una región en concreto. También señalan que el auge del movimiento independista obedece más a una manipulación política que a un deseo o necesidad real de los catalanes.

\subsection{La Vía Catalana}

Persigue la independencia de Cataluña y su configuración como Estado independiente y soberano. Es la opción defendida por los nacionalistas independentistas retratados en la foto 
del pasado 12 de diciembre. El término "Vía Catalana" es reciente y tiene su máximo exponente en la cadena humana formada en la Diada de 2013 con el fin de reivindicar gráficamente la independencia de Cataluña (la cadena humana simulaba recorrer los límites geográficos de la región). Esta propuesta está inspirada en la Vía Báltica, una cadena humana formada por Estonia, Letonia y Lituania en 1989 pidiendo la independencia frente a la antigua URSS, lo que consiguieron en 1991. Otras referencias recurrentes por parte de los independentistas son Escocia y Puerto Rico, la primera por el proceso que tiene lugar en estos momentos y el segundo por su estatus como Estado Libre Asociado de Estados Unidos. Ambos serán comentados más adelante.

En definitiva, frente a la opción del apartado siguiente -Tercera Vía- que contempla la posibilidad de una España federal, la Vía Catalana sólo aspira a la independencia.

\subsection{La Tercera Vía o federalismo}

La expresión "Tercera Vía” es acuñada por Josep Antoni Durán i Lleida, líder de Unió Democrática de Catalunya (parte de $\mathrm{CiU}$ ), y propone un camino intermedio entre la independencia y el status quo actual. El origen del término se encuentra en un artículo publicado por este político en La Vanguardia el 23 de septiembre de 2013, titulado "Ahora, consulta, ¿Después...?". Aunque esta "Tercera vía", en el momento de realizar el presente estudio, no se había materializado aún en propuestas concretas, "puede convertirse en un camino fructífero si se puede encontrar el punto de equilibrio entre las legítimas aspiraciones nacionales de Cataluña y los lazos efectivos y las diversas utilidades de todo tipo, resultado de muchos años de historia compartida, pero no siempre feliz" (Durán i Lleida, 2013). Es importante resaltar que la "Tercera vía" apoyaría la autodeterminación del pueblo catalán, y en ello coincide con la propuesta anterior, pero difiere en la forma en que dicha consulta debe ser realizada. La consulta que propugna Durán "se tiene que hacer de manera legal y con plena libertad para debatir todo lo que haga falta", en contraposición a la postura de Mas y sus socios, que defienden que la consulta debe hacerse por encima de todo y de todos.

\section{LA FACTIBILIDAD DE LA CONSULTA: ASPECTOS TÉCNICOS, JURÍDICOS Y POLÍTICOS}

En su comparecencia ante los medios de comunicación, el Presidente Mas indica que, con la fecha del 9 de noviembre, se da tiempo de sobra para que las instituciones del gobierno español puedan "negociar con las instituciones catalanas la forma de hacer esta consulta de acuerdo con los marcos legales existentes, que son muchos" (Comparecencia de Artur Mas frente a los medios el 12/12/2013. Minuto 02:00). Alude así Mas a la posibilidad técnica de realizar una consulta, negada siempre desde el gobierno central. Esta frase implica que si existe voluntad política, puede buscarse la vía para hacer una consulta legal.

Las dos estrategias que el gobierno de Mas decide poner en marcha son, por un lado, la petición formal desde el Parlamento catalán al Gobierno de España para que éste transfiera la capacidad de hacer referéndums a aquél en base a lo estipulado en el artículo 150.2 de la Constitución: "El Estado podrá transferir o delegar en las Comunidades Autónomas, mediante ley orgánica, facultades correspondientes a materia de titularidad estatal que por su propia naturaleza sean susceptibles de transferencia o delegación. La ley preverá en cada caso la correspondiente transferencia de medios financieros, así como las formas de control que se reserve el Estado". Esta es la opción que cuenta ya con una negativa por parte del gobierno, que no considera que la facultad de convocar un referéndum pro independentista 
sea una competencia trasferible a una región. Por otro, la opción de aprobar una Ley de consultas en el propio Parlamento catalán para posibilitar técnicamente la realización de la misma. A diferencia de un referéndum, el resultado de una "consulta" no es vinculante, es decir, no obliga a tomar ninguna decisión posterior. Ambas iniciativas fueron puestas en marcha en el momento de realizar este trabajo.

Es importante señalar en este punto que el Presidente del Gobierno Mariano Rajoy subraya que lo defendido por "algunos partidos políticos de Cataluña es radicalmente contrario a la Constitución y a la Ley" (Comparecencia de M. Rajoy, minuto 00:58). Concretamente, señala que "la iniciativa choca frontalmente con el fundamento mismo de la Constitución que es la indisoluble unidad de la nación española" (Comparecencia de M. Rajoy, minuto 01:08). El gobierno que preside Mariano Rajoy, según sus propias palabras, no puede negociar sobre algo que es "propiedad de todos los españoles" (Comparecencia de M. Rajoy, minuto 01:22).

Más allá de los planteamientos políticos la pregunta es, ¿existen o no vías legales y posibilidades técnicas para que se realice la consulta? Según el Instituto de Estudios Autonómicos de la Generalitat, hay hasta 5 maneras de encajar el referéndum separatista en el marco constitucional español (ver "Informe sobre los procedimientos legales a través de los que los ciudadanos y ciudadanas de Catalunya pueden ser consultados sobre su futuro político colectivo", IEA, p. 54: "el de la Ley catalana 4/2010 de consultas populares por vía de referéndum; los referéndums consultivos previstos en el artículo 92.1 CE; la delegación o transferencia de competencias del artículo 150.2 CE; los de la ley catalana de consultas no refrendarias que actualmente se tramita en el Parlamento y la reforma de la Constitución española para incorporar los referéndums consultivos de ámbito autonómico"). La que quieren utilizar sus promotores es la última: aquella por la cual el gobierno central podría ceder al regional, aplicando el artículo 150.2 de la Constitución, la competencia de convocar referéndums, que actualmente es competencia exclusiva del Estado. Esto ya fue intentado por el independentismo vasco (Plan Ibarretxe) y rechazado por el Congreso, y no se prevé en el caso catalán una suerte diferente (De la Nuez, 2012).

Técnicamente, el censo electoral sería necesario para realizar una consulta de este tipo, pero está en manos del gobierno central y no va a ser cedido al gobierno de Mas para este evento, por lo que la Ley de Consultas por vías distintas a los referéndums que está preparando el Parlamento Catalán propone la utilización del padrón municipal, además de permitir el voto desde los 16 años. Al no ser realizada la consulta utilizando el censo, sino el padrón municipal, el resultado no sería reconocido por las instituciones europeas, que sólo prevén "escuchar" aquellas consultas que se hayan realizado por la vía legal.

Una cuestión técnica de interés es también la interpretación misma del resultado de la consulta ya que, al tratarse de una doble pregunta, las combinaciones de respuesta son muchas. La doble pregunta es: “¿Quiere que Cataluña sea un Estado?” En caso afirmativo, el encuestado pasa a la siguiente: “QQuiere que ese Estado sea independiente?”. En una tabla elaborada por el GESOP (Gabinete de Estudios Sociales y de Opinión), organismo sobre el que volveremos más adelante, se muestran todas las posibles combinaciones de respuesta a la doble pregunta de la consulta.

Es decir, la consulta recogerá hasta 11 grupos de respuestas diferentes, que son las que se sitúan en la columna de la izquierda (siendo respuesta doble las del primer grupo), cuando una pregunta única hubiera arrojado 5 grupos: "Sí', "No", "en blanco", "no sabe" y "no contesta". Estos 5 grupos son más fáciles de analizar. En la pregunta pactada por el bloque nacionalista, no sólo hay más del doble de combinaciones, sino que surgen dudas respecto a 
cómo interpretar un "Sí, quiero que Cataluña sea un Estado" con un "No sabe" en la segunda parte de la pregunta. No todos estarían de acuerdo sobre a qué grupo sumarle estos indecisos...

Tabla 1: Posibles respuestas a la doble pregunta acordada para el referéndum catalán

\begin{tabular}{llccc}
\hline $\begin{array}{c}\text { ¿Quiere que } \\
\text { un estado? }\end{array}$ & $\begin{array}{c}\text { ¿Y quiere que } \\
\text { este estado sea } \\
\text { independiente? }\end{array}$ & $\begin{array}{c}\text { A } \\
\text { Total } \\
\text { muestra }\end{array}$ & $\begin{array}{c}\text { B } \\
\text { Sin } \\
\text { abstención }\end{array}$ & $\begin{array}{c}\text { Coto } \\
\text { Vocidido' } \\
\text { 'decidon }\end{array}$ \\
\hline Si & Si & 41,0 & 44,1 & 49,8 \\
Sí & No & 5,4 & 5,8 & 6,5 \\
Si & En blanco & 0,8 & 0,8 & 0,9 \\
Si & No sabe & 1,4 & 1,5 & \\
Sí & No contesta & 0,1 & 0,1 & \\
\hline No & & 28,3 & 30,4 & 34,3 \\
\hline En blanco & & 5,5 & 5,9 & 6,7 \\
Nulo & & 1,5 & 1,6 & 1,8 \\
Abstención & & 7,0 & & \\
No sabe & & 8,1 & 8,7 & \\
No contesta & & 1,0 & 1,1 & \\
\hline TOTAL & & 100,0 & 100,0 & 100,0 \\
\hline
\end{tabular}

Fuente: GESOP, en http://www.gesop.net/es/blog/item/157-la-consulta-i-les-enquestes

En este sentido, resulta de gran interés atender a lo que algunos expertos en la materia en el ámbito académico apuntan sobre esta cuestión. La cadena de radio catalanista RAC1 realizó el mismo 12 de diciembre un especial sobre la consulta de Mas. A lo largo de más de 5 horas de programa, se realizaron multitud de entrevistas tanto a los protagonistas del conflicto (referenciadas a lo largo de este trabajo en numerosas ocasiones) como a expertos en la materia desde el punto de vista jurídico.

A continuación se resumen las aportaciones de dos de ellos, que resultan de especial interés:

En primer lugar, en su entrevista para RAC1 (RAC1: 1212 17h min 4:02), Antonio Rovira Viñas, Catedrático de Derecho Constitucional de la Universidad Autónoma de Madrid, apunta las diferencias entre el proceso catalán y el escocés indicando, por ejemplo, que en el caso de Escocia no hay un código constitucional que cumplir (o incumplir), y lo que se inicia es una conversación para ver si hace falta más independencia, lo que no es el caso de Cataluña.

Este profesor apoya la realización de una consulta, e indica que procedimientos jurídicos constitucionales para llevarla a cabo efectivamente hay varios. En todo caso, lo fundamental es dar una salida a esta situación de enfrentamiento y para ello hay que entender que la Constitución no aprueba ni rechaza nada, sino que establece un marco jurídico para la convivencia. Nuestra Constitución recoge la posibilidad de convocar referéndums en su artículo 92 (Artículo 92: “1. Las decisiones políticas de especial trascendencia podrán ser sometidas a referéndum consultivo de todos los ciudadanos. 2. El referéndum será convocado por el Rey, mediante propuesta del Presidente del Gobierno, previamente autorizada por el 
Congreso de los Diputados. 3. Una ley orgánica regulará las condiciones y el procedimiento de las distintas modalidades de referéndum previstas en esta Constitución"), siendo este el único artículo que hace referencia a un proceso de consulta directa. Por su parte, el artículo 150.2 de la Constitución, como ya se ha visto, permitiría realizar una consulta sin salirse del marco legal, pero sin la voluntad política de actuar en el marco de este artículo (que, recordemos, permitía traspasar una competencia ostentada por el gobierno central a uno regional) es muy difícil que la consulta se realice. Si el Estado dice que no a todo, y no mantiene una vía de diálogo, la única forma es seguir adelante con la Ley de consultas proyectada por el Parlamento de Cataluña, aun sabiendo que el Tribunal Constitucional puede declararla anticonstitucional y dar al traste con todo. En este contexto, seguir adelante con el proceso -al margen del Estado y de la ley- sería prácticamente inviable por dos motivos:

1. En la práctica no es posible hacer un referéndum si el aparato administrativo del Estado no pone a disposición del gobierno catalán las juntas electorales, la policía, el censo electoral, el poder judicial, etc. A nivel internacional, si la consulta se realiza con medios privados (a través de la sociedad civil y sus organizaciones civiles o económicas, de carácter privado o mixto), sus resultados carecerían de reconocimiento y credibilidad.

2. Continuar con el proceso al margen del ordenamiento legal establecido podría dar lugar a actos que se consideran delictivos.

El parlamento de Cataluña, según este profesor, está haciendo lo que hay que hacer: solicitar formalmente la realización de una consulta. Pero si, como es previsible, esta vía no prospera, hacerla sin el consentimiento explícito o tácito del gobierno central no solo no es conveniente sino que es imposible (RAC1: 1212 17h min 11:45), subraya el profesor Rovira. La credibilidad -tanto interna como internacional - de una consulta pasa invariablemente por la utilización de la estructura política del Estado para su realización.

En segundo lugar, el Profesor Titular de Derecho Internacional Público de la Universidad de Barcelona, David Bondía (RAC1: 1212 17h min 16:00), señala que la comunidad internacional tan sólo reconoce el derecho a la autodeterminación de los pueblos en dos supuestos: colonialismo y violación de los derechos fundamentales de los ciudadanos por parte del propio Estado. Ninguno de los dos sucede en Cataluña por lo que, en principio, la comunidad internacional daría la espalda a la consulta. Existe la opción de ir por la vía de los hechos, es decir, hacer igualmente la consulta y con los resultados en la mano acudir a la comunidad internacional en busca de reconocimiento, como sucedió en Kosovo (no tenía tampoco la posibilidad en su ordenamiento jurídico interno de hacer un referéndum, pero lo hizo y al día siguiente declaró unilateralmente la independencia, logrando además el reconocimiento internacional). Esto es una solución "extrema" que tiene más riesgos que bondades, pero es una vía. Un riesgo claro es que no se obtenga el reconocimiento ya que la comunidad internacional, formada por Estados, no va a alentar movimientos de este tipo que atentan contra la integridad de los mismos.

Bondía, por otro lado, la pregunta planteada por el frente independentista no sólo es ilegal sino que está mal planteada, ya que no se pueden gestionar fácilmente las respuestas (sí-sí, síno, sí-abstención, sí-voto nulo, etc.). Para empezar, ¿a qué está respondiendo el ciudadano cuando dice que sí (o no) quiere que Cataluña sea un Estado? Es necesaria una labor pedagógica (no demagógica) intensa para explicar a la ciudadanía qué está votando exactamente en cada caso (RAC1: 1212 17h min 23:25). El profesor Bondía, por último, señala el error de una buena parte de los nacionalistas de tener como referente a Puerto Rico, Estado libre asociado de Estados Unidos, cuando las competencias que Cataluña tiene en 
estos momentos respecto del Estado Español son mucho mayores que las de Puerto Rico respecto a EEUU (RAC1: 1212 17h min 23:40).

Para finalizar, parece evidente a tenor de las intervenciones de los entrevistados, que uno de los aspectos más importantes que hay que cerrar durante este año es cómo se valida la consulta: ¿debe haber un porcentaje mínimo de participación ciudadana?, este porcentaje, ¿es suficiente con que sea absoluto o debe reflejar la distribución real de la población?, ¿cuándo se considera que el resultado ha sido positivo o negativo: ¿50\%+1 voto?, ¿qué grupos de respuesta serán consideradas favorables a la independencia y cuáles no? Esto último, que no sería necesario en caso de una única respuesta, es vital en el caso de la pregunta planteada ya que, como queda de manifiesto en la tabla elaborada por el GESOP, son demasiados los grupos de respuesta resultantes.

\section{CONCLUSIONES}

El "desafío independentista" planteado por la Generalitat de Cataluña sitúa al Gobierno español en una situación harto difícil. En un clima de absoluta confrontación, en el que partidarios y detractores de la consulta han dejado a un lado de facto la posibilidad de acuerdo, las únicas posibilidades para lograr una salida efectiva parecen ser:

(1) Seguir adelante con un proceso que no se ajusta al ordenamiento jurídico español y que, por tanto, se sitúa al margen de la legalidad, abriendo un escenario cuanto menos inquietante. El artículo 155 de la Constitución "permite al Gobierno, con la aprobación por mayoría absoluta del Senado, tomar las medidas oportunas en aras del interés general" entre las que estarían la proclamación del estado de sitio. Este movimiento de suspensión de las competencias autonómicas tendría como único antecedente a Manuel Azaña cuando, siendo Presidente de la II República, encarga al, por entonces, Presidente del Gobierno Juan Negrín, resolver la "cuestión catalana".

(2) La siguiente opción sería llevar a cabo una consulta "menor" y esgrimir sus resultados frente al gobierno español y frente a la comunidad internacional para lograr mayores cuotas de autonomía o incluso lograr ser un Estado federal. También podría verse este escenario como un paso más -un refuerzo- en el proceso hacia la independencia.

Más allá de cuestiones políticas o metodológicas, resulta preocupante, desde un punto de vista sociológico, la fractura social que se ha abierto no sólo entre los catalanes y el resto de españoles, sino entre los catalanes mismos. Distintas voces se alzan para denunciar que la tradicionalmente pacífica convivencia entre partidarios y detractores de la independencia se ha visto alterada por los acontecimientos de los últimos meses, de manera que se hace difícil vislumbrar un escenario en que dicha convivencia se recupere y que una vez finalizado el proceso, ocurra lo que ocurra, no hay ni vencedores ni vencidos. Tanto el mantenimiento del status quo actual como lograr la independencia de España se considerarían soluciones radicales por uno de los dos bandos de este conflicto. Quizá esa Tercera Vía que apunta Durán i Lleida tendría más posibilidades de lograr un consenso, aunque dejara inicialmente a personas descontentas a ambos lados.

Aun es pronto para saber qué va a ocurrir en adelante. Los próximos meses serán vitales para conocer hacia cuál de los escenarios o vías descritas se avanza en Cataluña. 


\section{BIBLIOGRAFÍA}

ALDEGUER, B.; ROMERO, A. y BELMONTE, I. (2013). "La opinión pública española y las pautas de apoyo a la democracia en un contexto de crisis económica". XI Congreso Español de Sociología. BALCELLS, A. (2003). Breve historia del nacionalismo catalán. Madrid: Alianza Editorial. CANAL, J. (coord.) (2006). El nacionalismo catalán: mitos y lugares de memoria. Biblioteca Nueva. CINCO DÍAS (2013). "Van Rompuy: La UE no aplicaría los tratados a Cataluña si declara la independencia" en https://www.youtube.com/watch?v=WHsFuPruKYA [consulta 13/12/2013] CONSTITUCIÓN ESPAÑOLA (1978). BOE, n 311, 29 de diciembre 1978, pp. 29313-29424.

CONVIVENCIA CÍVICA CATALANA (4 diciembre 2013). “¿Son fiables las encuestas del gobierno catalán?" en http://bit.ly/1mvqILz [consulta 13/12/2013]

CRÓNICA GLOBAL (17 enero 2014). "Rosa Díez se pregunta "a qué está esperando" Rajoy para intervenir la autonomía de Catalunya", en http://bit.ly/Pr6qXn [consulta 19/1/2014]

CRUZ, M.C. (2013). "Mas pacta la consulta soberanista: "¿Quiere que Cataluña sea un Estado?" e "¿independiente?" en RTVE, http://bit.ly/1gPmb1K [consulta 19/12/2013]

DE LA NUEZ, E. (10 de octubre 2012). "El Estado de papel" en El Mundo Orbyt y recuperado del blog Hay Derecho en http://bit.ly/1jwnqDM [consulta 19/1/2014]

DURÁN I LLEIDA, J. A. (23 septiembre 2012). “¡Ahora, consulta!, ¿Después...?” en La Vanguardia http://bit.ly/RJcWLd [consulta 6/1/2014]

EL MUNDO (13 diciembre 2013). "El artículo 155 de la Constitución”, en http://mun.do/1cDIOqH [consulta 27/12/2013]

EL MUNDO (5 noviembre 2012). "Intelectuales y sociedad civil se movilizan contra la ola independentista en Cataluña", en http://mun.do/1mvqcNv [Consulta: $2 / 1 / 2014]$

EL PERIÓDICO (13 diciembre 2013). "Camacho asegura que Mas "ha dinamitado los puentes de diálogo con el Gobierno"”, en http://bit.ly/11B7fHV [consulta 27/12/2013]

EUROPA PRESS (2013). "Rajoy: "La consulta catalana es inconstitucional"' canal Youtube de Europa Press en http://bit.ly/1icJJ69 [consulta 19/12/2013]

GARCÍA, R. (2002). De los elogios a Felipe V. Madrid, Centro de Estudios Políticos y Constitucionales.

GRUPO RAMÓN LLULL (17 de febrero de 2011). "Cataluña, la decepción de Azaña", en Diario de Mallorca, http://bit.ly/1flYCaQ [consulta 10/2/2014]

INSTITUT D'ESTUDIS AUTONOMICS GENERALITAT DE CATALUNYA (2013). "Informe sobre los procedimientos legales a través de los que los ciudadanos y ciudadanas de Catalunya pueden ser consultados sobre su futuro político colectivo", en http://bit.ly/1k4gG30 [consulta 8/1/2014]

LA VANGUARDIA (2013). "Mas anuncia los términos de la consulta soberanista" vídeo editado por Atlas en http://bit.ly/1OXKhJQ [consulta 18/12/2013]

MEDINA, F. (24 octubre 2013). "El gran negocio de la independencia", en El Plural digital http://bit.ly/1jFDxjJ [consulta 20/12/2013]

MOLINA, I. (6 diciembre 2012). "Kosovo y Cataluña: distintos y distantes". Elcano Blog, Spanish foreign affairs, en http://bit.ly/QCZRSv [consulta 19/12/2013]

ORGANIZACIÓN INTERNACIONAL PARA LAS MIGRACIONES (OIM) (2010). Impactos de la crisis sobre la población inmigrante, en http://bit.ly/1gPlq95 [consulta 10/1/2014]

RICO, J. (14 diciembre 2013). "El 44\% de catalanes votarían por la independencia y el 36\%, en contra", en El Periódico digital http://bit.ly/1rfXi6c [consulta 14/12/2013]

ROGER, M. (14 septiembre 2013). "La guardiana del independentismo", en El País digital, http://bit.ly/1hlFFdH [consulta 20/12/2013]

ROIG, J. (1998). El nacionalismo catalán (1800-1939). Barcelona: Arcos Libros.

SANZ, L. A. (6 noviembre 2012). "El alto coste de la independencia", en El Mundo digital http://mun.do/1tyrX0v [consulta 20/12/2013] 
RADIO ON LINE: archivos de audio (podcats) del programa Versió RAC1 de Radio Associació de Catalunya 1.

"18:00 12-12-2013 - Dijous. VERSIO RAC1" (duración 60 minutos). Archivo de audio del Programa Versió RAC 1, disponible en línea en http://rac1.org/a-la-carta/

"17:00 12-12-2013 - Dijous. VERSIO RAC1" (duración 61 minutos). Archivo de audio del Programa Versió RAC 1, disponible en línea en http://rac1.org/a-la-carta/

"16:00 12-12-2013 - Dijous. VERSIO RAC1" (duración 60 minutos). Archivo de audio del Programa Versió RAC 1, disponible en línea en http://rac1.org/a-la-carta/

\section{Breve currículo:}

\section{Elena Llorca Asensi}

Licenciada en Sociología por la Universidad de Alicante y Máster en Comunicación Digital por la Universidad Miguel Hernández de Elche. Desde junio de 2013 colabora con el Área de Ciencia Política y de la Administración de la Universidad de Alicante, donde aporta su visión de la Administración Pública y el Liderazgo político tras más de una década de trabajar para el sector público en algunas de las fundaciones del gobierno valenciano. Ha colaborado en varias publicaciones relacionadas con la modernización de la Administración Pública y la democracia electrónica. 